MATHEMATICS OF COMPUTATION

Volume 81, Number 278, April 2012, Pages 1005-1023

S $0025-5718(2011) 02520-2$

Article electronically published on July 26, 2011

\title{
THE GENERATING FUNCTION FOR THE DIRICHLET SERIES $L_{m}(s)$
}

\author{
WILLIAM Y. C. CHEN, NEIL J. Y. FAN, AND JEFFREY Y. T. JIA
}

\begin{abstract}
The Dirichlet series $L_{m}(s)$ are of fundamental importance in number theory. Shanks defined the generalized Euler and class numbers in connection with the Dirichlet series, denoted by $\left\{s_{m, n}\right\}_{n \geq 0}$. We obtain a formula for the exponential generating function $s_{m}(x)$ of $s_{m, n}$, where $m$ is an arbitrary positive integer. In particular, for $m>1$, say, $m=b u^{2}$, where $b$ is square-free and $u>1$, we show that $s_{m}(x)$ can be expressed as a linear combination of the four functions $w(b, t) \sec (b t x)( \pm \cos ((b-p) t x) \pm \sin (p t x))$, where $p$ is a nonnegative integer not exceeding $b, t \mid u^{2}$ and $w(b, t)=K_{b} t / u$ with $K_{b}$ being a constant depending on $b$. Moreover, the Dirichlet series $L_{m}(s)$ can be easily computed from the generating function formula for $s_{m}(x)$. Finally, we show that the main ingredient in the formula for $s_{m, n}$ has a combinatorial interpretation in terms of the $\Lambda$-alternating augmented $m$-signed permutations defined by Ehrenborg and Readdy. More precisely, when $m$ is square-free, this answers a question posed by Shanks concerning a combinatorial interpretation of the numbers $s_{m, n}$. When $m$ is not square-free, say $m=b u^{2}$, the numbers $K_{b}^{-1} s_{m, n}$ can be written as a linear combination of the numbers of $\Lambda$-alternating augmented $b t$-signed permutations with integer coefficients, where $t \mid u^{2}$.
\end{abstract}

\section{INTRODUCTION}

The Dirichlet series

$$
L_{m}(s)=\sum_{\substack{l>0 \\ \text { odd } l}}\left(\frac{-m}{l}\right) \frac{1}{l^{s}},
$$

where $(-m / l)$ is the Jacobi symbol, originate in the distribution of primes into arithmetic progressions, the class number of binary quadratic forms, as well as the distribution of the Legendre and Jacobi symbols. They play a crucial role in the computation of certain number-theoretic constants; see [3, 6, 12, 14. Several approaches have been developed for the computation of $L_{m}(s)$; see, for example, Shanks [15, 16, 18.

The generalized Euler and class numbers were introduced by Shanks for the computation of the Dirichlet series $L_{m}(s)$ [15, 17. These numbers are also related to derivative polynomials and Euler polynomials; see Hoffman [7] and Shanks [17.

Received by the editor April 13, 2010 and, in revised form, December 26, 2010.

2010 Mathematics Subject Classification. Primary 11B68, 05 A05.

Key words and phrases. Dirichlet series, generalized Euler and class number, $\Lambda$-alternating augmented $m$-signed permutation, $r$-cubical lattice, Springer number.

(C)2011 American Mathematical Society Reverts to public domain 28 years from publication 
In this paper, we obtain the generating functions for the generalized Euler and class numbers. Let us recall the definition of the generalized Euler and class numbers $s_{m, n}(m \geq 1, n \geq 0)$, introduced by Shanks,

$$
s_{m, n}= \begin{cases}c_{m, \frac{n}{2}} & \text { if } n \text { is even, } \\ d_{m, \frac{n+1}{2}} & \text { if } n \text { is odd, }\end{cases}
$$

where $c_{m, n}$ and $d_{m, n}$ are given by

$$
\begin{gathered}
c_{m, n}=(2 n) ! L_{m}(2 n+1)\left(K_{m} \sqrt{m}\right)^{-1}\left(\frac{\pi}{2 m}\right)^{-2 n-1}, \\
d_{m, n}=(2 n-1) ! L_{-m}(2 n)\left(K_{m} \sqrt{m}\right)^{-1}\left(\frac{\pi}{2 m}\right)^{-2 n},
\end{gathered}
$$

in which

$$
K_{m}= \begin{cases}\frac{1}{2} & \text { if } m=1 \\ 1 & \text { otherwise }\end{cases}
$$

Set

$$
\begin{aligned}
c_{m}(x) & =\sum_{n \geq 0} c_{m, n} \frac{x^{2 n}}{(2 n) !}, \\
d_{m}(x) & =\sum_{n \geq 1} d_{m, n} \frac{x^{2 n-1}}{(2 n-1) !}, \\
s_{m}(x) & =\sum_{n \geq 0} s_{m, n} \frac{x^{n}}{n !} .
\end{aligned}
$$

Clearly,

$$
s_{m}(x)=c_{m}(x)+d_{m}(x) .
$$

By the definitions (1.2) and (1.3), we have

$$
\begin{aligned}
& \sum_{n \geq 0} L_{m}(2 n+1) x^{2 n}=\frac{K_{m} \pi \sqrt{m}}{2 m} c_{m}\left(\frac{\pi}{2 m} x\right), \\
& \sum_{n \geq 1} L_{-m}(2 n) x^{2 n-1}=\frac{K_{m} \pi \sqrt{m}}{2 m} d_{m}\left(\frac{\pi}{2 m} x\right) .
\end{aligned}
$$

Therefore, if we set

$$
\hat{L}_{m}(s)= \begin{cases}L_{-m}(s+1) & \text { if } s \text { is odd } \\ L_{m}(s+1) & \text { if } s \text { is even }\end{cases}
$$

then

$$
\sum_{s \geq 0} \hat{L}_{m}(s) x^{s}=\frac{K_{m} \pi \sqrt{m}}{2 m} s_{m}\left(\frac{\pi}{2 m} x\right) .
$$

It follows from (1.5) that $\hat{L}_{m}(s)$ is determined by $s_{m}(x)$. In other words, the generating function $s_{m}(x)$ leads to a quick way to compute $\hat{L}_{m}(s)$.

Consider $c_{m, n}$ and $d_{m, n}$ as entries of the infinite matrices $C$ and $D$, respectively. Then the first column of $C$ forms the sequence of class numbers in connection with primitive binary quadratic forms, and the first row of $C$ forms the sequence of secant numbers, corresponding to up-down permutations, also called alternating 
permutations, of even length. Meanwhile, the first row of $D$ forms the sequence of tangent numbers corresponding to up-down permutations of odd length. Recall that both secant numbers and tangent numbers are called Euler numbers. This is why the numbers $s_{m, n}$ are called generalized Euler and class numbers.

Shanks [17 found recurrence relations for $c_{m, n}$ and $d_{m, n}$ with respect to the index $n$, from which it follows that $c_{m, n}$ and $d_{m, n}$ are integers. For example, we have

$$
\sum_{i=0}^{n}(-4)^{i}\left(\begin{array}{c}
2 n \\
2 i
\end{array}\right) c_{2, n-i}=(-1)^{n}
$$

and

$$
\sum_{i=0}^{n-1}(-4)^{i}\left(\begin{array}{c}
2 n-1 \\
2 i
\end{array}\right) d_{2, n-i}=(-1)^{n-1}
$$

In fact, due to the well-known Euler product of the Dirichlet series $L_{m}(s)$ (see [8, 11]), it can be easily shown that $c_{m, n}$ and $d_{m, n}$ are positive.

Shanks [17] raised the question of whether the generalized Euler and class numbers may have some combinatorial interpretations. The combinatorial interpretations of $s_{m, n}$ for $m=1,2,3,4$, have been found. Let $\left(s_{m, n}\right)_{n \geq 0}$ denote the conjoined sequence

$$
\left(c_{m, 0}, d_{m, 1}, c_{m, 1}, d_{m, 2}, c_{m, 2}, d_{m, 3}, \ldots\right) \text {. }
$$

For $m=1$, the sequence $(1,1,1,2,5,16, \ldots)$ is listed as A000111 in Sloane [19], which is called the sequence of Euler numbers, enumerating alternating permutations on $[n]=\{1,2, \ldots, n\}$.

For $m=2$, the sequence $(1,1,3,11,57,361, \ldots)$ is numbered $A 001586$ in [19], which is also called the sequence of Springer numbers which arise in the work of Springer on the theory of Weyl groups.

For $m=3$, the sequence $(1,2,8,46,352,3362, \ldots)$ is listed as A007289 in [19], and we call it the sequence of Ehrenborg and Readdy numbers. The combinatorial interpretation of this sequence in terms of alternating 3-signed permutations has been given by Ehrenborg and Readdy [4].

For $m=4$, a combinatorial interpretation of the sequence $(1,4,16,128,1280$, $16384, \ldots)$ has been given implicitly by Ehrenborg and Readdy [5] in terms of nonaugmented André $R$-signed permutations on $[n]$ with $R=(4,4, \ldots, 4)$.

For $m \geq 5$, we shall give formulas for the generating function for $s_{m}(x)$. For the case when $m$ is square-free, we shall give a combinatorial interpretation for $s_{m, n}$. In the general case, we see that the number $s_{m, n}$ or $2 s_{m, n}$ is a linear combination with integer coefficients of some numbers with a combinatorial interpretation.

For $m=1,2,3,4$, it is known that

$$
\begin{aligned}
& s_{1}(x)=\sec x+\tan x, \\
& s_{2}(x)=\frac{\cos x+\sin x}{\cos 2 x}, \\
& s_{3}(x)=\frac{\sin 2 x+\cos x}{\cos 3 x}, \\
& s_{4}(x)=\sec 4 x+\tan 4 x .
\end{aligned}
$$


From our formulas, we get the following expressions for $m=5,6,7$ :

$$
\begin{aligned}
& s_{5}(x)=\frac{\cos 4 x+\sin x}{\cos 5 x}+\frac{\cos 2 x+\sin 3 x}{\cos 5 x}, \\
& s_{6}(x)=\frac{\cos 5 x+\sin x}{\cos 6 x}+\frac{\cos x+\sin 5 x}{\cos 6 x}, \\
& s_{7}(x)=\frac{\cos 3 x+\sin 4 x}{\cos 7 x}+\frac{\cos x+\sin 6 x}{\cos 7 x}-\frac{\cos 5 x+\sin 2 x}{\cos 7 x} .
\end{aligned}
$$

This paper is organized as follows. In Section 2 we compute the generating function $s_{m}(x)$ when $m$ is square-free, while in Section 3 we consider the case when $m$ is not square-free. Section 4 is devoted to the combinatorial interpretation of the numbers $s_{m, n}$ in terms of $m$-signed permutations as introduced by Ehrenborg and Readdy when $m$ is square-free.

\section{Computation For $s_{m}(x)$ When $m$ IS SQUARE-FReE}

In this section, we compute the generating function $s_{m}(x)$ when $m>1$ and $m$ is square-free. For $0 \leq p \leq m$, we adopt the following notation introduced in [4,

$$
\Lambda_{m, p}(x):=\frac{\cos ((m-p) x)+\sin (p x)}{\cos (m x)} .
$$

When $m$ is square-free, we shall not encounter the case that $m$ is a multiple of 4 . We shall have three formulas for $s_{m}(x)$ depending on the residue of $m$ modulo 4.

Theorem 2.1. Assume that $m$ is square-free and $m=4 t+3$. Then

$$
s_{m}(x)=\sum_{k=1}^{t}\left(\frac{k}{m}\right) \Lambda_{m, 4 k}(x)+\sum_{k=t+1}^{2 t+1}\left(\frac{k}{m}\right) \Lambda_{m, 2 m-4 k}(x) .
$$

Theorem 2.2. Assume that $m$ is square-free and $m=4 t+1$. Then

$$
s_{m}(x)=\sum_{k=1}^{t}\left(\frac{k}{m}\right) \Lambda_{m, m-4 k}(x)-\sum_{k=t+1}^{2 t}\left(\frac{k}{m}\right) \Lambda_{m, 4 k-m}(x) .
$$

Theorem 2.3. Assume that $m$ is square-free and $m=4 t+2$. Then

$$
s_{m}(x)=\sum_{\substack{k=1 \\ \text { odd } k}}^{4 t+1}\left(\frac{-m}{k}\right) \Lambda_{m, k}(x) .
$$

To prove the above theorems, let us first recall the following formula of $L_{m}(2 n+1)$ obtained by Shanks [15, 17.

Lemma 2.4. Suppose that $m>1$ and $m$ is square-free. Then $L_{m}(2 n+1)$ can be expressed as a linear combination of the Fourier series $S_{2 n+1}(x)$. More precisely,

$$
L_{m}(2 n+1)=\frac{2}{\sqrt{m}} \sum_{k} \epsilon_{k} S_{2 n+1}\left(y_{k}\right)
$$

where the Jacobi symbols $\epsilon_{k}$ and rational numbers $y_{k}$ are uniquely determined by $m$, and $S_{2 n+1}(x)$ is defined by

$$
S_{2 n+1}(x)=\sum_{k=0}^{\infty} \frac{\sin 2 \pi(2 k+1) x}{(2 k+1)^{2 n+1}} .
$$


Furthermore, we have

$$
c_{m}(x)=\frac{1}{\cos (m x)} \sum_{k} \epsilon_{k} \cos \left(m x\left(1-4 y_{k}\right)\right) .
$$

In fact, Shanks has given an explicit procedure to determine the constants $\epsilon_{k}$ and $y_{k}$. To compute $\epsilon_{k}$ and $y_{k}$, we use the definition (1.1) of the series $L_{m}(s)$ and express the Jacobi symbol $\left(\frac{-m}{l}\right)$ as a linear combination of sines according to the following expansion; see 9 .

Proposition 2.5. Assume that $l$ is odd and $m$ satisfies the following two conditions: $m \equiv 1(\bmod 4)$ or $m \equiv 8$ or $12(\bmod 16)$ and $p^{2} \nmid m$ for any odd prime $p$. Then we have

$$
\left(\frac{m}{l}\right)=\frac{1}{\sqrt{m}} \sum_{r=1}^{|m|}\left(\frac{m}{r}\right) e^{2 \pi i l r /|m|} .
$$

In particular, when $m \equiv 3(\bmod 4)$, we have $-m \equiv 1(\bmod 4)$ and we can use the above expansion for $\left(\frac{-m}{l}\right)$. Similarly, when $m \equiv 1(\bmod 4)$, we see that $-4 m \equiv 12(\bmod 16)$ so that we can compute $\left(\frac{-4 m}{l}\right)$ by using the above formula. Finally, when $m \equiv 2(\bmod 4)$, we find $-4 m \equiv 8(\bmod 16)$ so that we can compute $\left(\frac{-4 m}{l}\right)$. Note that when $l$ is odd, we have

$$
\left(\frac{-4 m}{l}\right)=\left(\frac{-m}{l}\right) \text {. }
$$

Thus, the Jacobi symbol $\left(\frac{-m}{l}\right)$ can be determined by the above procedure for $m>1$.

On the other hand, Shanks [15, 17] provided the following formula for $L_{-m}(2 n)$.

Lemma 2.6. Suppose that $m>1$ and $m$ is square-free. Then $L_{-m}(2 n)$ can be expressed as a linear combination of the Fourier series $C_{2 n}(x)$. To be more specific,

$$
L_{-m}(2 n)=\frac{2}{\sqrt{m}} \sum_{k} \epsilon_{k}^{\prime} C_{2 n}\left(y_{k}^{\prime}\right),
$$

where the Jacobi symbols $\epsilon_{k}^{\prime}$ and rational numbers $y_{k}^{\prime}$ are uniquely determined by $m$, and $C_{2 n}(x)$ is defined by

$$
C_{2 n}(x)=\sum_{k=0}^{\infty} \frac{\cos 2 \pi(2 k+1) x}{(2 k+1)^{2 n}}
$$

Moreover,

$$
d_{m}(x)=\frac{1}{\cos (m x)} \sum_{k} \epsilon_{k}^{\prime} \sin \left(m x\left(1-4 y_{k}^{\prime}\right)\right) .
$$

Similarly, Shanks has shown how to compute the constants $\epsilon_{k}^{\prime}$ and $y_{k}^{\prime}$. In order to compute $\epsilon_{k}^{\prime}$ and $y_{k}^{\prime}$, recall that

$$
L_{-m}(s)=\sum_{\substack{l>0 \\ \text { odd } l}}\left(\frac{m}{l}\right) \frac{1}{l^{s}},
$$

where the Jacobi symbol $\left(\frac{m}{l}\right)$ can be expressed as a linear combination of cosines, resorting to Proposition 2.5. 
For the case $m \equiv 3(\bmod 4)$, we find $4 m \equiv 12(\bmod 16)$ so that we can use the above expansion for $\left(\frac{4 m}{l}\right)$. When $m \equiv 1(\bmod 4)$, we can also compute $\left(\frac{m}{l}\right)$ by using the above expansion. Finally, when $m \equiv 2(\bmod 4)$, we see that $4 m \equiv$ $8(\bmod 16)$ and $\left(\frac{4 m}{l}\right)$ can be determined in the same manner. Note that when $l$ is odd, the following relation holds:

$$
\left(\frac{4 m}{l}\right)=\left(\frac{m}{l}\right) \text {. }
$$

Thus, the Jacobi symbol $\left(\frac{m}{l}\right)$ can be determined for $m>1$.

Keep in mind that $m$ is assumed to be square-free. Set

$$
\hat{c}_{m}(x)=\cos (m x) c_{m}(x), \quad \hat{d}_{m}(x)=\cos (m x) d_{m}(x), \quad \hat{s}_{m}(x)=\cos (m x) s_{m}(x) .
$$

Proof of Theorem 2.1. Since $m \equiv 3(\bmod 4)$, by using the expansion (2.10) for $\left(\frac{-m}{l}\right)$ and $\left(\frac{4 m}{l}\right)$, we have

$$
\epsilon_{k}=\left(\frac{k}{m}\right), \quad y_{k}=\frac{k}{m}, \quad \epsilon_{k}^{\prime}=\left(\frac{m}{k}\right), \quad y_{k}^{\prime}=\frac{k}{4 m} .
$$

Thus,

$$
\begin{aligned}
L_{m}(2 n+1) & =\frac{2}{\sqrt{m}} \sum_{k=1}^{(m-1) / 2}\left(\frac{k}{m}\right) S_{2 n+1}\left(\frac{k}{m}\right), \\
L_{-m}(2 n) & =\frac{2}{\sqrt{m}} \sum_{\text {odd } k<m}\left(\frac{m}{k}\right) C_{2 n}\left(\frac{k}{4 m}\right) .
\end{aligned}
$$

Therefore, by Lemmas 2.4 and 2.6 we obtain

$$
\begin{aligned}
\hat{s}_{m}(x)=\hat{c}_{m}(x)+\hat{d}_{m}(x)= & \sum_{k=1}^{(m-1) / 2}\left(\frac{k}{m}\right) \cos (m-4 k) x \\
& +\sum_{\text {odd } k<m}\left(\frac{m}{k}\right) \sin (m-k) x .
\end{aligned}
$$

Suppose that $m=4 t+3$. It follows that

$$
\begin{aligned}
\hat{c}_{m}(x) & =\sum_{k=1}^{t}\left(\frac{k}{m}\right) \cos (m-4 k) x+\sum_{k=t+1}^{2 t+1}\left(\frac{k}{m}\right) \cos (m-4 k) x \\
& =\sum_{k=1}^{t}\left(\frac{k}{m}\right) \cos (m-4 k) x+\sum_{k=t+1}^{2 t+1}\left(\frac{k}{m}\right) \cos (4 k-m) x \\
\hat{d}_{m}(x) & =\sum_{\substack{k=1 \\
\text { odd } k}}^{4 t+1}\left(\frac{m}{k}\right) \sin (m-k) x
\end{aligned}
$$

Thus, we obtain

$$
\begin{aligned}
\hat{s}_{m}(x)= & \sum_{k=1}^{t}\left(\left(\frac{k}{m}\right) \cos (m-4 k) x+\left(\frac{m}{m-4 k}\right) \sin (4 k) x\right) \\
& +\sum_{k=t+1}^{2 t+1}\left(\left(\frac{k}{m}\right) \cos (4 k-m) x+\left(\frac{m}{4 k-m}\right) \sin (2 m-4 k) x\right) .
\end{aligned}
$$


It remains to verify that

$$
\left(\frac{k}{m}\right)=\left(\frac{m}{m-4 k}\right)
$$

for $1 \leq k \leq t$ and

$$
\left(\frac{k}{m}\right)=\left(\frac{m}{4 k-m}\right)
$$

for $t+1 \leq k \leq 2 t+1$.

As for 2.11), since both $m$ and $m-4 k$ are odd positive numbers, if they are coprime, then by the law of quadratic reciprocity, we find

$$
\begin{aligned}
\left(\frac{m}{m-4 k}\right) & =-\left(\frac{m-4 k}{m}\right)=-\left(\frac{-4 k}{m}\right)=-\left(\frac{-4}{m}\right)\left(\frac{k}{m}\right) \\
& =-\left(\frac{-1}{m}\right)\left(\frac{4}{m}\right)\left(\frac{k}{m}\right)=\left(\frac{2}{m}\right)^{2}\left(\frac{k}{m}\right)=\left(\frac{k}{m}\right) .
\end{aligned}
$$

Otherwise, suppose that the greatest common divisor of $m$ and $m-4 k$ is greater than 1, i.e., $(m, m-4 k)>1$. From the definition of the Jacobi symbol, it is clear that

We wish to show that

$$
\left(\frac{m}{m-4 k}\right)=0
$$

$$
\left(\frac{k}{m}\right)=0
$$

Since $(m, m-4 k)|m,(m, m-4 k)|(m-4 k)$ and $m-(m-4 k)=4 k$, we deduce that

$$
(m, m-4 k) \mid 4 k \text {. }
$$

But the fact that $m$ is odd implies that $((m, m-4 k), 4)=1$. Thus from (2.13) it follows that $(m, m-4 k) \mid k$. So we conclude that $(m, m-4 k) \mid(m, k)$ and $(m, k)>1$, which yields

$$
\left(\frac{k}{m}\right)=\left(\frac{m}{m-4 k}\right)=0
$$

Similarly, (2.12) can be checked via the following steps if $4 k-m$ and $m$ are coprime,

$$
\begin{aligned}
\left(\frac{m}{4 k-m}\right) & =\left(\frac{4 k-m}{m}\right)=\left(\frac{-(m-4 k)}{m}\right)=\left(\frac{-1}{m}\right)\left(\frac{m-4 k}{m}\right) \\
& =-\left(\frac{m-4 k}{m}\right)=-\left(\frac{-4 k}{m}\right)=\left(\frac{k}{m}\right) .
\end{aligned}
$$

For the case $(m, 4 k-m)>1$, by the definition of the Jacobi symbol, it is evident that

In this case, we claim that

$$
\left(\frac{m}{4 k-m}\right)=0 .
$$

$$
\left(\frac{k}{m}\right)=0 .
$$

Since $(m, 4 k-m)|m,(m, 4 k-m)|(4 k-m)$ and $m+(4 k-m)=4 k$, we deduce that

$$
(m, 4 k-m) \mid 4 k \text {. }
$$


Because $m$ is odd, we see that $((m, 4 k-m), 4)=1$. Thus from (2.14) it follows that $(m, 4 k-m) \mid k$. Therefore, we obtain that $(m, 4 k-m) \mid(m, k)$ and $(m, k)>1$, which implies

$$
\left(\frac{k}{m}\right)=\left(\frac{m}{4 k-m}\right)=0
$$

So we find that

$$
\begin{aligned}
s_{m}(x)= & \sum_{k=1}^{t}\left(\frac{k}{m}\right) \frac{\cos (m-4 k) x+\sin (4 k) x}{\cos m x} \\
& +\sum_{k=t+1}^{2 t+1}\left(\frac{k}{m}\right) \frac{\cos (4 k-m) x+\sin (2 m-4 k) x}{\cos m x} .
\end{aligned}
$$

This completes the proof.

Proof of Theorem 2.2, Under the condition $m \equiv 1(\bmod 4)$, by applying the expansion (2.10) to $\left(\frac{-4 m}{l}\right)$ and $\left(\frac{m}{l}\right)$, we get

$$
\epsilon_{k}=\left(\frac{-m}{k}\right), \quad y_{k}=\frac{k}{4 m}, \quad \epsilon_{k}^{\prime}=\left(\frac{k}{m}\right), \quad y_{k}^{\prime}=\frac{k}{m} .
$$

It follows that

$$
\begin{aligned}
L_{m}(2 n+1) & =\frac{2}{\sqrt{m}} \sum_{\text {odd } k<m}\left(\frac{-m}{k}\right) S_{2 n+1}\left(\frac{k}{4 m}\right), \\
L_{-m}(2 n) & =\frac{2}{\sqrt{m}} \sum_{k=1}^{(m-1) / 2}\left(\frac{k}{m}\right) C_{2 n}\left(\frac{k}{m}\right) .
\end{aligned}
$$

In view of Lemmas 2.4 and 2.6 we find

$$
\hat{s}_{m}(x)=\hat{c}_{m}(x)+\hat{d}_{m}(x)=\sum_{\text {odd } k<m}\left(\frac{-m}{k}\right) \cos (m-k) x+\sum_{k=1}^{(m-1) / 2}\left(\frac{k}{m}\right) \sin (m-4 k) x .
$$

Writing $m=4 t+1$, we obtain

$$
\begin{aligned}
\hat{d}_{m}(x) & =\sum_{k=1}^{t}\left(\frac{k}{m}\right) \sin (m-4 k) x+\sum_{k=t+1}^{2 t}\left(\frac{k}{m}\right) \sin (m-4 k) x \\
& =\sum_{k=1}^{t}\left(\frac{k}{m}\right) \sin (m-4 k) x-\sum_{k=t+1}^{2 t}\left(\frac{k}{m}\right) \sin (4 k-m) x, \\
\hat{c}_{m}(x) & =\sum_{\substack{k=1 \\
\text { odd } k}}^{4 t-1}\left(\frac{-m}{k}\right) \cos (m-k) x .
\end{aligned}
$$

Consequently,

$$
\begin{aligned}
\hat{s}_{m}(x)= & \sum_{k=1}^{t}\left(\left(\frac{k}{m}\right) \sin (m-4 k) x+\left(-\frac{m}{m-4 k}\right) \cos (4 k) x\right) \\
& +\sum_{k=t+1}^{2 t}\left(-\left(\frac{k}{m}\right) \sin (4 k-m) x+\left(-\frac{m}{4 k-m}\right) \cos (2 m-4 k) x\right) .
\end{aligned}
$$


Finally, we need to show that

$$
\left(\frac{k}{m}\right)=\left(-\frac{m}{m-4 k}\right)
$$

for $1 \leq k \leq t$ and

$$
-\left(\frac{k}{m}\right)=\left(-\frac{m}{4 k-m}\right)
$$

for $t+1 \leq k \leq 2 t$.

To verify (2.15), we consider two cases. If $(m, m-4 k)=1$, since both $m$ and $m-4 k$ are odd, we may employ the law of quadratic reciprocity to deduce that

$$
\begin{aligned}
\left(-\frac{m}{m-4 k}\right) & =\left(\frac{-1}{m-4 k}\right)\left(\frac{m}{m-4 k}\right)=\left(\frac{m}{m-4 k}\right)=\left(\frac{m-4 k}{m}\right)=\left(\frac{-4 k}{m}\right) \\
& =\left(\frac{-4}{m}\right)\left(\frac{k}{m}\right)=\left(\frac{-1}{m}\right)\left(\frac{2}{m}\right)^{2}\left(\frac{k}{m}\right)=\left(\frac{k}{m}\right) .
\end{aligned}
$$

For the case $(m, m-4 k)>1$, by definition,

$$
\left(-\frac{m}{m-4 k}\right)=0
$$

We wish to show that

$$
\left(\frac{k}{m}\right)=0
$$

Since $(m, m-4 k)|m,(m, m-4 k)|(m-4 k)$ and $m-(m-4 k)=4 k$, we get

$$
(m, m-4 k) \mid 4 k \text {. }
$$

However, the fact that $m$ is odd implies that $((m, m-4 k), 4)=1$. Thus, from (2.17) we see that $(m, m-4 k) \mid k$. It follows that $(m, m-4 k) \mid(m, k)$ and $(m, k)>1$. Hence

$$
\left(\frac{k}{m}\right)=\left(-\frac{m}{m-4 k}\right)=0 .
$$

To prove (2.16), we also consider two cases. If $m$ and $4 k-m$ are coprime, then we have

$$
\begin{aligned}
-\left(-\frac{m}{4 k-m}\right) & =-\left(\frac{-1}{4 k-m}\right)\left(\frac{m}{4 k-m}\right)=\left(\frac{m}{4 k-m}\right)=\left(\frac{4 k-m}{m}\right) \\
& =\left(\frac{-(m-4 k)}{m}\right)=\left(\frac{-1}{m}\right)\left(\frac{m-4 k}{m}\right)=\left(\frac{-4 k}{m}\right)=\left(\frac{k}{m}\right) .
\end{aligned}
$$

If $(m, 4 k-m)>1$, then we get

$$
-\left(-\frac{m}{4 k-m}\right)=0
$$

We need to show that

$$
\left(\frac{k}{m}\right)=0
$$

Since $(m, 4 k-m)|m,(m, 4 k-m)|(4 k-m)$ and $m+(4 k-m)=4 k$, we find

$$
(m, 4 k-m) \mid 4 k \text {. }
$$


But $m$ is odd, we see that $((m, 4 k-m), 4)=1$. From (2.18) it follows that $(m, 4 k-m) \mid k$. This implies that $(m, 4 k-m) \mid(m, k)$ and $(m, k)>1$. Consequently,

$$
\left(\frac{k}{m}\right)=-\left(-\frac{m}{4 k-m}\right)=0 \text {. }
$$

Thus we obtain

$$
\begin{aligned}
s_{m}(x)= & \sum_{k=1}^{t}\left(\frac{k}{m}\right) \frac{\cos (4 k) x+\sin (m-4 k) x}{\cos m x} \\
& -\sum_{k=t+1}^{2 t}\left(\frac{k}{m}\right) \frac{\cos (2 m-4 k) x+\sin (4 k-m) x}{\cos m x} .
\end{aligned}
$$

This completes the proof.

Proof of Theorem 2.3. Since $m \equiv 2(\bmod 4)$, by using the expansion (2.10) for $\left(\frac{-4 m}{l}\right)$ and $\left(\frac{4 m}{l}\right)$, we obtain

$$
\epsilon_{k}=\left(\frac{-m}{k}\right), \quad y_{k}=\frac{k}{4 m}, \quad \epsilon_{k}^{\prime}=\left(\frac{m}{k}\right), \quad y_{k}^{\prime}=\frac{k}{4 m} .
$$

Hence

$$
\begin{aligned}
L_{m}(2 n+1) & =\frac{2}{\sqrt{m}} \sum_{\text {odd } k<m}\left(\frac{-m}{k}\right) S_{2 n+1}\left(\frac{k}{4 m}\right), \\
L_{-m}(2 n) & =\frac{2}{\sqrt{m}} \sum_{\text {odd } k<m}\left(\frac{m}{k}\right) C_{2 n}\left(\frac{k}{4 m}\right) .
\end{aligned}
$$

By Lemmas 2.4 and 2.6, we see that

$$
\hat{s}_{m}(x)=\hat{c}_{m}(x)+\hat{d}_{m}(x)=\sum_{\text {odd } k<m}\left(\frac{-m}{k}\right) \cos (m-k) x+\sum_{\text {odd } k<m}\left(\frac{m}{k}\right) \sin (m-k) x .
$$

Writing $m=4 t+2$, since

$$
\left(\frac{4 t+2}{2 t+1}\right)=0
$$

we obtain

$$
\hat{s}_{m}(x)=\sum_{\substack{k=1 \\ \text { odd } k}}^{4 t+1}\left(\left(\frac{-m}{k}\right) \cos (m-k) x+\left(\frac{m}{m-k}\right) \sin (k) x\right) .
$$

Finally, it follows from $-k \equiv m-k(\bmod m)$ that

$$
\left(\frac{-m}{k}\right)=\left(\frac{m}{m-k}\right)
$$

for $1 \leq k \leq 4 t+1$. Hence we conclude that

$$
s_{m}(x)=\sum_{\substack{k=1 \\ \text { odd } k}}^{4 t+1}\left(\frac{-m}{k}\right) \frac{\cos (m-k) x+\sin (k) x}{\cos m x} .
$$

This completes the proof.

For $m=5,6,7$, the generating functions $s_{m}(x)$ have been given in the Introduction. 


\section{Computation for $s_{m}(x)$ When $m$ is not Square-FreE}

In this section, we obtain an expression for $s_{m}(x)$ for the case when $m$ is not square-free. Assume that $m$ can be divided by a square $u^{2}$ and $u>1$. Recall that the constant $K_{b}$ is given by (1.4).

Theorem 3.1. Suppose that $m=b u^{2}$, where $b$ is square-free and $u>1$. Then we can express $s_{m}(x)$ as a linear combination of the four functions

$$
w(b, t) \sec (b t x)( \pm \cos ((b-p) t x) \pm \sin (p t x)),
$$

where $p$ is a nonnegative integer not exceeding $b, t \mid u^{2}$ and $w(b, t)=K_{b} t / u$.

The idea of the proof is to establish two recursive relations (3.21) and 3.22) between $s_{m, n}$ and $s_{b, n}$. Then we express $s_{m}(x)$ as a linear combination of the functions $c_{b}(t x)$ and $d_{b}(t x)$ by considering the two cases according to whether there exist odd prime factors $u_{i}$ of $u$ with residues 3 modulo 4 . Since $b$ is square-free, $c_{b}(t x)$ and $d_{b}(t x)$ can be evaluated by using the formulas in the previous section.

Proof. Let us start with the following relation given by Shanks 15

$$
L_{m}(s)=L_{b}(s) \prod_{u_{i} \mid u}\left(1-\left(\frac{-b}{u_{i}}\right) \frac{1}{u_{i}^{s}}\right)
$$

where the product ranges over odd primes $u_{i}$ (if any) that divide $u$. To be precise, in case there are no odd prime factors, the empty product is defined to be 1 . From the definitions (1.2) and (1.3) it follows that

$$
\begin{aligned}
& c_{m, n}=K_{b} u\left(u^{2}\right)^{2 n} \prod_{i}\left(u_{i}^{2 n+1}-\left(\frac{-b}{u_{i}}\right)\right)\left(\prod_{i} \frac{1}{u_{i}}\right)^{2 n+1} c_{b, n}, \\
& d_{m, n}=K_{b} u\left(u^{2}\right)^{2 n-1} \prod_{i}\left(u_{i}^{2 n}-\left(\frac{b}{u_{i}}\right)\right)\left(\prod_{i} \frac{1}{u_{i}}\right)^{2 n} d_{b, n} .
\end{aligned}
$$

For the purpose of computing $s_{m}(x)$ for the case when $m$ is not square-free, we need to consider two cases according to whether there exist $u_{i} \equiv 3(\bmod 4)$ among the $k$ odd factors $u_{1}, u_{2}, \ldots, u_{k}$ of $u$.

Case 1. $u_{i} \equiv 1(\bmod 4)$ for $1 \leq i \leq k$. In this case, we see that

$$
\left(\frac{-b}{u_{i}}\right)=\left(\frac{b}{u_{i}}\right) \text {. }
$$

Suppose that among the factors $u_{1}, u_{2}, \ldots, u_{k}$ there are $k_{1}$ primes $u_{1}, u_{2}, \ldots, u_{k_{1}}$ satisfying $\left(\frac{b}{u_{i}}\right)=1$ for $1 \leq i \leq k_{1}, k_{2}$ primes $u_{k_{1}+1}, u_{k_{1}+2}, \ldots, u_{k_{1}+k_{2}}$ satisfying $\left(\frac{b}{u_{k_{1}+j}}\right)=-1$ for $1 \leq j \leq k_{2}$, and $k_{3}$ primes $u_{k_{1}+k_{2}+1}, u_{k_{1}+k_{2}+2}, \ldots, u_{k_{1}+k_{2}+k_{3}}$ satisfying $\left(\frac{b}{u_{k_{1}+k_{2}+l}}\right)=0$ for $1 \leq l \leq k_{3}$, where $k_{1}+k_{2}+k_{3}=k$. From (3.21) it is clear that

$$
c_{m, n}=K_{b} u\left(u^{2}\right)^{2 n} \frac{\prod_{i=1}^{k_{1}}\left(u_{i}^{2 n+1}-1\right) \times \prod_{j=1}^{k_{2}}\left(u_{k_{1}+j}^{2 n+1}+1\right)}{\prod_{i=1}^{k_{1}+k_{2}} u_{i}^{2 n+1}} c_{b, n} .
$$


Let

$$
f_{c}=\frac{\prod_{i=1}^{k_{1}}\left(u_{i}^{2 n+1}-1\right) \times \prod_{j=1}^{k_{2}}\left(u_{k_{1}+j}^{2 n+1}+1\right)}{\prod_{i=1}^{k_{1}+k_{2}} u_{i}^{2 n+1}} .
$$

In this notation, (3.23) can be rewritten as

$$
c_{m, n}=K_{b} u\left(u^{2}\right)^{2 n} f_{c} c_{b, n},
$$

which implies that

$$
c_{m}(x)=K_{b} u \sum_{n \geq 0} f_{c} c_{b, n} \frac{\left(u^{2} x\right)^{2 n}}{(2 n) !} .
$$

Since

$$
\prod_{i=1}^{k_{1}}\left(u_{i}^{2 n+1}-1\right)=\prod_{i=1}^{k_{1}} u_{i}^{2 n+1}-\sum_{i=1}^{k_{1}}\left(\prod u_{1} \cdots u_{i-1} u_{i+1} \cdots u_{k_{1}}\right)^{2 n+1}+\cdots+(-1)^{k_{1}}
$$

and

$$
\begin{aligned}
\prod_{j=1}^{k_{2}}\left(u_{k_{1}+j}^{2 n+1}+1\right)= & \prod_{j=1}^{k_{2}} u_{k_{1}+j}^{2 n+1} \\
& +\sum_{j=1}^{k_{2}}\left(\prod u_{k_{1}+1} \ldots u_{k_{1}+j-1} u_{k_{1}+j+1} \cdots u_{k_{1}+k_{2}}\right)^{2 n+1}+\cdots+1
\end{aligned}
$$

we can expand $f_{c}$ as follows

$$
\begin{aligned}
f_{c}=1 & +\sum_{j=1}^{k_{2}} \frac{1}{u_{k_{1}+j}^{2 n+1}}+\cdots+\frac{1}{\prod_{j=1}^{k_{2}} u_{k_{1}+j}^{2 n+1}}-\sum_{i=1}^{k_{1}} \frac{1}{u_{i}^{2 n+1}}-\sum_{i=1}^{k_{1}} \sum_{j=1}^{k_{2}} \frac{1}{u_{i}^{2 n+1} u_{k_{1}+j}^{2 n+1}}-\cdots \\
& -\sum_{i=1}^{k_{1}} \frac{1}{u_{i}^{2 n+1} \prod_{j=1}^{k_{2}} u_{k_{1}+j}^{2 n+1}}+\cdots+\frac{(-1)^{k_{1}}}{\prod_{i=1}^{k_{1}+k_{2}} u_{i}^{2 n+1}} .
\end{aligned}
$$

Plugging (3.25) into (3.24), we find that $c_{m}(x)$ is a linear combination of the functions $c_{b}(t x)$, where $t \mid u^{2}$ and the coefficient of $c_{b}(t x)$ equals $K_{b} t / u$.

Similarly, we have

$$
d_{m, n}=K_{b} u\left(u^{2}\right)^{2 n-1} \frac{\prod_{i=1}^{k_{1}}\left(u_{i}^{2 n}-1\right) \times \prod_{j=1}^{k_{2}}\left(u_{k_{1}+j}^{2 n}+1\right)}{\prod_{i=1}^{k_{1}+k_{2}} u_{i}^{2 n}} d_{b, n} .
$$

Let

$$
f_{d}=\frac{\prod_{i=1}^{k_{1}}\left(u_{i}^{2 n}-1\right) \times \prod_{j=1}^{k_{2}}\left(u_{k_{1}+j}^{2 n}+1\right)}{\prod_{i=1}^{k_{1}+k_{2}} u_{i}^{2 n}},
$$

then (3.26) can be rewritten as

$$
d_{m, n}=K_{b} u\left(u^{2}\right)^{2 n-1} f_{d} d_{b, n},
$$


which leads to the relation

$$
d_{m}(x)=K_{b} u \sum_{n \geq 1} f_{d} d_{b, n} \frac{\left(u^{2} x\right)^{2 n-1}}{(2 n-1) !} .
$$

Again, we may expand $f_{d}$ as follows:

$$
\begin{aligned}
f_{d}= & +\sum_{j=1}^{k_{2}} \frac{1}{u_{k_{1}+j}^{2 n}}+\cdots+\frac{1}{\prod_{j=1}^{k_{2}} u_{k_{1}+j}^{2 n}}-\sum_{i=1}^{k_{1}} \frac{1}{u_{i}^{2 n}}-\sum_{i=1}^{k_{1}} \sum_{j=1}^{k_{2}} \frac{1}{u_{i}^{2 n} u_{k_{1}+j}^{2 n}}-\cdots \\
& -\sum_{i=1}^{k_{1}} \frac{1}{u_{i}^{2 n} \prod_{j=1}^{k_{2}} u_{k_{1}+j}^{2 n}}+\cdots+\frac{(-1)^{k_{1}}}{\prod_{i=1}^{k_{1}+k_{2}} u_{i}^{2 n}} .
\end{aligned}
$$

Substituting (3.28) into (3.27), we find that $d_{m}(x)$ is a linear combination of the functions $d_{b}(t x)$, where $t \mid u^{2}$ and the coefficient of $d_{b}(t x)$ equals $K_{b} t / u$. Furthermore, we see that $c_{m}(x)$ and $d_{m}(x)$ have the same coefficients for the linear combinations. In other words, the relation for $c_{m}(x)$ and $c_{b}(t x)$ is still valid after changing $c_{m}(x)$ and $c_{b}(t x)$ to $d_{m}(x)$ and $d_{b}(t x)$, respectively. Therefore, in this case, $s_{m}(x)$ can be expressed as a sum of the functions $w(b, t)\left(c_{b}(t x)+d_{b}(t x)\right)$, where $t \mid u^{2}$ and $w(b, t)=K_{b} t / u$.

Case 2. Among the $k$ primes $u_{1}, u_{2}, \ldots, u_{k}$, there exist $q$ primes $u_{i_{1}}, u_{i_{2}}, \ldots, u_{i_{q}}$ with residue 3 modulo 4 . To compute $s_{m}(x)$, we first consider the case when $q=1$, and then argue that the case $q>1$ can be dealt with in the same way.

Since $q=1$, we assume that the first $k-1$ odd primes $u_{1}, u_{2}, \ldots, u_{k-1}$ satisfy $u_{i} \equiv 1(\bmod 4)$ for $1 \leq i \leq k-1$, and suppose that the last prime $u_{k}$ satisfies $u_{k} \equiv 3(\bmod 4)$, or

$$
\left(\frac{-b}{u_{k}}\right)=-\left(\frac{b}{u_{k}}\right)
$$

We now define the indices $k_{1}, k_{2}$ and $k_{3}$ as in Case 1 except that $k_{1}+k_{2}+k_{3}=k-1$ since there are $k-1$ primes with residue 1 modulo 4 . This leads us to consider two subcases according to whether $\left(\frac{-b}{u_{k}}\right)$ equals 0 . Keep in mind that we have $q=1$ in these two subcases.

On one hand, in order to use the two recursive relations (3.21) and (3.22) between $s_{m, n}$ and $s_{b, n}$, we may assume that $\left(\frac{-b}{u_{k}}\right)=0$. Therefore, the term $u_{k}$ on the denominator and the same term on the numerator cancel each other in (3.21). This argument also applies to the relation (3.22). In other words, there is no need to consider the occurrence of the term $u_{k}^{2 n+1}$ in (3.21) and the term $u_{k}^{2 n}$ in (3.22). In this sense, it remains to consider the other $k-1$ primes $u_{1}, u_{2}, \ldots, u_{k-1}$ such that $u_{i} \equiv 1(\bmod 4)$ for $1 \leq i \leq k-1$. By the argument in Case 1 , we see again that $s_{m}(x)$ can be expressed as a sum of the terms $w(b, t)\left(c_{b}(t x)+d_{b}(t x)\right)$, where $t \mid u^{2}$ and $w(b, t)=K_{b} t / u$.

On the other hand, we should consider the case when $\left(\frac{-b}{u_{k}}\right)=1$ or $\left(\frac{-b}{u_{k}}\right)=-1$. Since the proofs for these two cases are similar, we only give the proof for the case 
$\left(\frac{-b}{u_{k}}\right)=-1$. From 3.21 it follows that

$$
\begin{aligned}
c_{m, n}=K_{b} u\left(u^{2}\right)^{2 n} \frac{\prod_{i=1}^{k_{1}}\left(u_{i}^{2 n+1}-1\right) \prod_{j=1}^{k_{2}}\left(u_{k_{1}+j}^{2 n+1}+1\right)}{\prod_{i=1}^{k_{1}+k_{2}} u_{i}^{2 n+1}} \times \frac{u_{k}^{2 n+1}+1}{u_{k}^{2 n+1}} c_{b, n} \\
=K_{b} u\left(u^{2}\right)^{2 n}\left(\frac{\prod_{i=1}^{k_{1}}\left(u_{i}^{2 n+1}-1\right) \prod_{j=1}^{k_{2}}\left(u_{k_{1}+j}^{2 n+1}+1\right)}{\prod_{i=1}^{k_{1}+k_{2}} u_{i}^{2 n+1}}\right. \\
\left.+\frac{\prod_{i=1}^{k_{1}}\left(u_{i}^{2 n+1}-1\right) \prod_{j=1}^{k_{2}}\left(u_{k_{1}+j}^{2 n+1}+1\right)}{u_{k}^{2 n+1} \prod_{i=1}^{k_{1}+k_{2}} u_{i}^{2 n+1}}\right) c_{b, n} .
\end{aligned}
$$

Let

$$
g_{b}(x)=K_{b} u \sum_{n \geq 0}\left(\frac{\prod_{i=1}^{k_{1}}\left(u_{i}^{2 n+1}-1\right) \prod_{j=1}^{k_{2}}\left(u_{k_{1}+j}^{2 n+1}+1\right)}{\prod_{i=1}^{k_{1}+k_{2}} u_{i}^{2 n+1}}\right) c_{b, n} \frac{\left(u^{2} x\right)^{2 n}}{(2 n) !} .
$$

Then we have

$$
c_{m}(x)=g_{b}(x)+\frac{1}{u_{k}} g_{b}\left(x / u_{k}\right) .
$$

In fact, by the argument in Case 1 , we see that $g_{b}(x)$ is a linear combination of the functions $c_{b}(t x)$, where $t \mid u^{2}$ and the coefficient of $c_{b}(t x)$ equals $K_{b} t / u$.

Similarly, by 3.22 we find

$$
\begin{aligned}
d_{m, n}=K_{b} u\left(u^{2}\right)^{2 n-1} \frac{\prod_{i=1}^{k_{1}}\left(u_{i}^{2 n}-1\right) \prod_{j=1}^{k_{2}}\left(u_{k_{1}+j}^{2 n}+1\right)}{\prod_{i=1}^{k_{1}+k_{2}} u_{i}^{2 n}} \times \frac{u_{k}^{2 n}-1}{u_{k}^{2 n}} d_{b, n} \\
=K_{b} u\left(u^{2}\right)^{2 n-1}\left(\frac{\prod_{i=1}^{k_{1}}\left(u_{i}^{2 n}-1\right) \prod_{j=1}^{k_{2}}\left(u_{k_{1}+j}^{2 n}+1\right)}{\prod_{i=1}^{k_{1}+k_{2}} u_{i}^{2 n}}\right. \\
\left.-\frac{\prod_{i=1}^{k_{1}}\left(u_{i}^{2 n}-1\right) \prod_{j=1}^{k_{2}}\left(u_{k_{1}+j}^{2 n}+1\right)}{u_{k}^{2 n} \prod_{i=1}^{k_{1}+k_{2}} u_{i}^{2 n}}\right) d_{b, n} .
\end{aligned}
$$

Let

$$
h_{b}(x)=K_{b} u \sum_{n \geq 1}\left(\frac{\prod_{i=1}^{k_{1}}\left(u_{i}^{2 n}-1\right) \prod_{j=1}^{k_{2}}\left(u_{k_{1}+j}^{2 n}+1\right)}{\prod_{i=1}^{k_{1}+k_{2}} u_{i}^{2 n}}\right) d_{b, n} \frac{\left(u^{2} x\right)^{2 n-1}}{(2 n-1) !} .
$$

We get

$$
d_{m}(x)=h_{b}(x)-\frac{1}{u_{k}} h_{b}\left(x / u_{k}\right) .
$$

Again, from the reasoning in Case 1 it follows that $h_{b}(x)$ is a linear combination of the functions $d_{b}(t x)$, where $t \mid u^{2}$ and the coefficient of $d_{b}(t x)$ equals $K_{b} t / u$. Combining the equations (3.29) and (3.30) yields the following relation:

$$
s_{m}(x)=g_{b}(x)+h_{b}(x)+\frac{1}{u_{k}}\left(g_{b}\left(x / u_{k}\right)-h_{b}\left(x / u_{k}\right)\right) .
$$

Thus we obtain that $s_{m}(x)$ can also be expressed as a linear combination of the functions $w(b, t)\left( \pm c_{b}(t x) \pm d_{b}(t x)\right)$, where $t \mid u^{2}$ and $w(b, t)=K_{b} t / u$.

Finally, as mentioned before we shall show that the justification for the above two subcases can be applied to the case for $q>1$. Let us give an example for $q=2$. Suppose that $u_{k-1}$ and $u_{k}$ are the last two primes such that $u_{k-1} \equiv 3(\bmod 4)$, 
$u_{k} \equiv 3(\bmod 4)$, and $\left(\frac{-b}{u_{k-1}}\right)=-1,\left(\frac{-b}{u_{k}}\right)=-1$. Using the functions $g_{b}(x)$ and $h_{b}(x)$ as given before, we can deduce the following formula for $s_{m}(x)$ :

$$
\begin{aligned}
s_{m}(x)= & g_{b}(x)+h_{b}(x)+\frac{1}{u_{k-1}}\left(g_{b}\left(x / u_{k-1}\right)-h_{b}\left(x / u_{k-1}\right)\right) \\
& +\frac{1}{u_{k}}\left(g_{b}\left(x / u_{k}\right)-h_{b}\left(x / u_{k}\right)\right) \\
& +\frac{1}{u_{k-1} u_{k}}\left(g_{b}\left(x / u_{k-1} u_{k}\right)+h_{b}\left(x / u_{k-1} u_{k}\right)\right) .
\end{aligned}
$$

Clearly, now $s_{m}(x)$ can also be written as a linear combination of the functions $w(b, t)\left( \pm c_{b}(t x) \pm d_{b}(t x)\right)$, where $t \mid u^{2}$ and $w(b, t)=K_{b} t / u$. For the other conditions on $u_{k-1}$ and $u_{k}$, the computation can be done in the same spirit.

In summary, $s_{m}(x)$ can be expressed as a linear combination of the functions $w(b, t)\left( \pm c_{b}(t x) \pm d_{b}(t x)\right)$, where $t \mid u^{2}$ and $w(b, t)=K_{b} t / u$. Since $b$ is squarefree, $c_{b}(t x)$ and $d_{b}(t x)$ can be written as a linear combination of the functions $\sec (b t x) \cos ((b-p) t x)$ and $\sec (b t x) \sin (p t x)$, respectively, by using the formulas in the previous section. This completes the proof.

Here we give three examples corresponding to the above three cases. For Case 1 , suppose that $m=3(5 \times 13)^{2}=3(65)^{2}=3 \times 4225=12675$. Then we have

$$
s_{12675}(x)=65 s_{3}(4225 x)-5 s_{3}(325 x)+13 s_{3}(845 x)-s_{3}(65 x),
$$

where

$$
s_{3}(x)=\sec (3 x)(\sin 2 x+\cos x) .
$$

For the first subcase of Case 2, suppose that $m=6(5 \times 3)^{2}=3(15)^{2}=6 \times 225=$ 1350. Then we get

$$
s_{1350}(x)=15 s_{6}(225 x)-3 s_{6}(45 x),
$$

where

$$
s_{6}(x)=\sec (6 x)(\cos 5 x+\sin x)+\sec (6 x)(\cos x+\sin 5 x) .
$$

For the second subcase of Case 2, assume that $m=225=(5 \times 3)^{2}$. We find

$$
\begin{aligned}
2 s_{225}(x)= & 15\left(c_{1}(225 x)+d_{1}(225 x)\right)-3\left(c_{1}(45 x)+d_{1}(45 x)\right) \\
& +5\left(c_{1}(75 x)-d_{1}(75 x)\right)-\left(c_{1}(15 x)-d_{1}(15 x)\right),
\end{aligned}
$$

where

$$
c_{1}(x)=\sec x, \quad d_{1}(x)=\tan x
$$

\section{A Combinatorial interpretation For $s_{m, n}$}

In this section, we aim to give a combinatorial interpretation of $s_{m, n}$ when $m$ is square-free based on the generating function formula for $s_{m}(x)$. Let us recall the known combinatorial interpretations of $s_{m, n}$ when $m=1,2,3,4$.

For $m=1,\left(s_{1, n}\right)_{n \geq 0}$ is called the sequence of Euler numbers. Let $E_{n}$ be the $n$-th Euler number, that is, the number of up-down permutations on $[n]=\{1,2, \ldots, n\}$, which are also called snakes of type $A_{n-1}$ by Arnol'd [2]. The following generating function is due to André [1]:

$$
\sum_{n \geq 0} E_{n} \frac{x^{n}}{n !}=\sec x+\tan x .
$$


Note that Springer also gave an explanation of the Euler numbers in terms of the irreducible root system $A_{n-1}$ and derived the generating function of André in this context.

For $m=2$, the sequence $\left(s_{2, n}\right)_{n \geq 0}$ turns out to be the sequence of Springer numbers of the irreducible root system $B_{n}$ [20. Purtill [13] has found an interpretation of this sequence. Let $P_{n}$ be the $n$-th entry of this sequence, whereas Purtill used the notation $E_{n}^{ \pm}$. He has shown that $P_{n}$ equals the number of André signed permutations on $[n]$. On the other hand, it has been shown by Arnol'd [2 that $S_{n}$ also counts the number of snakes of type $B_{n}$. Hoffman [7] has derived the generating function of the number of snakes of type $B_{n}$ by giving a direct combinatorial proof.

For $m=3$, the sequence $\left(s_{3, n}\right)_{n>0}$ has been studied by Ehrenborg and Readdy 4. Let $F_{n}$ denote the $n$-th Ehrenborg and Readdy number, which was denoted by $\left|E R_{n}\right|$; see Hoffman [7]. It has been shown that $F_{n}$ equals the number $\Lambda$-alternating augmented 3-signed permutations on [n]. Meanwhile, Hoffman [7] presented another combinatorial interpretation of the sequence in the case $m=3$ in terms of $E R_{n^{-}}$ snakes in the spirit of the snakes of type $A_{n-1}$ and of type $B_{n}$.

For $m=4$, the sequence $\left(s_{4, n}\right)_{n \geq 0}$ has also been studied by Ehrenborg and Readdy [5. p. 719]. In fact, they introduced the concept of nonaugmented André $R$-signed permutations on a finite set $N$ with $|N|=n$, where $R=\left(r_{i}\right)_{i \in N}$ is a vector indexed by $N$. They have shown that the exponential generating function for the number of nonaugmented André $R$-signed permutations with the $n$-tuple $R=(r, r, \ldots, r)$ is $\sec (r x)+\tan (r x)$. Therefore, for the $n$-tuple $R=(4,4, \ldots, 4)$, it follows that the number of nonaugmented André $R$-signed permutations is counted by $s_{4, n}$.

We proceed to give a combinatorial interpretation for $s_{m, n}$ when $m$ is square-free based on the generating function $s_{m}(x)$. More precisely, in this case we shall show that $s_{m}(x)$ can be expressed by the generating function $\Lambda_{m, p}(x)$ for the numbers $\Lambda_{m, p, n}$ defined by Ehrenborg and Readdy [4]. Consequently, when $m$ is square-free, the numbers $s_{m, n}$ can be written as a linear combination of the numbers $\Lambda_{m, p, n}$ with coefficients being 1 or -1 . With the aid of the combinatorial interpretation of the generating function due to Ehrenborg and Readdy, we see that $s_{m, n}$ can be explained in terms of maximal chains in the $m$-cubical lattice.

When $m$ is not square-free, write $m=b u^{2}$. The numbers $s_{m, n}$ or $2 s_{m, n}$ can be written as a linear combination of the numbers $\Lambda_{b t, p t, n}$ with integer coefficients, where $t \mid u^{2}$ and $0 \leq p \leq b$. However, these integer coefficients are obtained from the generating function of $s_{m}(x)$. It would be interesting to find a combinatorial explanation of these coefficients when $m$ is not square-free.

We now need some definitions. Ehrenborg and Readdy [4 defined a class of posets called the Sheffer posets, which can be viewed as a generalization of the binomial posets introduced by Stanley [21. As an important example, they studied the $r$-cubical lattice, which is a set of ordered $r$-tuples $\left(A_{1}, A_{2}, \ldots, A_{r}\right)$ of subsets from an infinite set $I$ together with the reverse inclusion order and with a minimum element $\hat{0}$ adjoined. Note that the $r$-cubical lattice has been studied by Metropolis, Rota, Strehl and White [10. Ehrenborg and Readdy further generalized the concept of $R$-labelings to linear edge-labelings. By considering the set of maximal chains in the interval $[\hat{0}, \hat{1}]$ on the Hasse diagram of the $r$-cubical lattice, they derived a formula for the number of $\Lambda$-alternating augmented $r$-signed permutations. 
To describe the definition of $\Lambda$-alternating augmented $r$-signed permutations, Ehrenborg and Readdy constructed a linear edge-labeling on the Hasse diagram of the $r$-cubical lattice. To be more specific, for an edge corresponding to the cover relation $A<B$ with $A \neq \hat{0}$, let $(i, j)$ denote its label where $i$ equals the unique index such that $A_{i} \neq B_{i}$ and $j$ is the singleton element in $A_{i}-B_{i}$. Let $G$ be the label of the edge corresponding to $\hat{0}<A$, which is called the special element by Ehrenborg and Readdy. Then an augmented $r$-signed permutation is a list $\left(G,\left(i_{1}, j_{1}\right),\left(i_{2}, j_{2}\right), \ldots,\left(i_{n}, j_{n}\right)\right)$, where $i_{1}, i_{2}, \ldots, i_{n} \in[r]$, and $\left(j_{1}, j_{2}, \ldots, j_{n}\right)$ forms a permutation on $[n]$. In other words, $r$-signed permutations are permutations on $[n]$ in which each element is assigned one of the $r$ signs.

To define the descent set of $r$-signed permutations, let $\Lambda$ be the set of such labels of the edges on the Hasse diagram of the $r$-cubical lattice. As is easily seen,

$$
\Lambda=([r] \times[n]) \cup\{G\} .
$$

Let $p$ be an integer such that $0 \leq p \leq r$. For fixed positive integers $r, n$ and $p$, we can define a linear order on $\Lambda$ which satisfies the conditions

$$
(i, j)<_{\Lambda} G \Rightarrow i \leq r-p
$$

and

$$
(i, j)>_{\Lambda} G \Rightarrow i>r-p,
$$

where $(i, j)$ is the label of the edge corresponding to the cover relation $A<B$ such that $A$ covers $\hat{0}$, and $G$ is the special element. For the remaining labels, we may arrange them in the lexicographic order. The descent set of an augmented $r$-signed permutation $\left(g_{0}, g_{1}, \ldots, g_{n}\right)$ is defined as the set $\left\{k: g_{k-1}>_{\Lambda} g_{k}\right\}$, where $g_{0}=G$ and $g_{k}=\left(i_{k}, j_{k}\right)$ for $1 \leq k \leq n$. Therefore, for an $\Lambda$-alternating augmented $r$-signed permutation, that is, permutation having descent set $\{2,4,6, \ldots\}$, it is necessary to have the condition $\left(i_{1}, j_{1}\right)>_{\Lambda} G$, or $i_{1}>r-p$. In other words, the labels above $G$ in this ordering are those whose first coordinate belongs to the set $\{r-p+1, r-p+2, \ldots, r\}$.

Recall that $\Lambda_{r, p, n}$ denotes the number of $\Lambda$-alternating augmented $r$-signed permutations. Since there is a one-to-one correspondence between the set of maximal chains in the $r$-cubical lattice and the set of augmented $r$-signed permutations, $\Lambda_{r, p, n}$ also equals the number of maximal chains with descent set $\{2,4,6, \ldots\}$ in the $r$-cubical lattice for which the first nonspecial edge has label $\left(i_{1}, j_{1}\right)$ with $r-p+1 \leq i_{1} \leq r$. Here the first nonspecial edge is defined as the edge corresponding to the cover relation $A<B$ such that $A$ covers $\hat{0}$. Based on this observation, Ehrenborg and Readdy derived the following generating function for $\Lambda_{r, p, n}$ :

$$
\Lambda_{r, p}(x)=\sum_{n \geq 0} \Lambda_{r, p, n} \frac{x^{n}}{n !}=\frac{\cos ((r-p) x)+\sin (p x)}{\cos (r x)} .
$$

In view of the above formula (4.33) and the generating functions for the generalized Euler and class numbers $s_{m, n}$ in the previous sections, for any square-free $m$ we can give a combinatorial interpretation of $s_{m, n}$ in terms of the augmented $m$ signed permutations, or equivalently, in terms of the maximal chains in the Hasse diagram of the $m$-cubical lattice.

When $m$ is square-free, as is shown in Section $2, s_{m, n}$ is a linear combination of the numbers $\Lambda_{m, p, n}$ with coefficients being 1 or -1 . For example, when $m=7$, 
since

$$
s_{7}(x)=\frac{\cos 3 x+\sin 4 x}{\cos 7 x}+\frac{\cos x+\sin 6 x}{\cos 7 x}-\frac{\cos 5 x+\sin 2 x}{\cos 7 x},
$$

we obtain that

$$
s_{7, n}=\Lambda_{7,4, n}+\Lambda_{7,6, n}-\Lambda_{7,2, n} .
$$

When $m$ is not square-free, assume that $m=b u^{2}$ and $u>1$. By Theorem (3.1), $K_{b}^{-1} s_{m, n}$ can be written as a linear combination of the numbers $\Lambda_{b t, p t, n}$ with integer coefficients. Recall that $K_{1}^{-1}=2$ and $K_{b}^{-1}=1$ for $b \neq 1$. For instance, we have

$$
2 s_{9}(x)=3(\sec (9 x)+\tan (9 x))+(\sec (3 x)-\tan (3 x)),
$$

which implies

$$
2 s_{9, n}=3 \Lambda_{9,9, n}+(-1)^{n} \Lambda_{3,3, n} .
$$

\section{ACKNOWLEDGMENTS}

We wish to thank the referee for valuable suggestions. This work was supported by the 973 Project, the PCSIRT Project of the Ministry of Education, and the National Science Foundation of China.

\section{REFERENCES}

[1] D. André, Sur les permutations alternées, J. Math. Pures Appl. 7 (1881), 167-184.

[2] V.I. Arnol'd, The calculus of snakes and the combinatorics of Bernoulli, Euler, and Springer numbers of Coxeter groups, Uspekhi Mat. Nauk. 47 (1992), 3-45 (Russian); Russian Math. Surveys 47 (1992), 1-51. MR 1171862 (93h:20042)

[3] P.T. Bateman and R.A. Horn, A heuristic asymptotic formula concerning the distribution of prime numbers, Math. Comp. 16 (1962), 363-367. MR0148632 (26:6139)

[4] R. Ehrenborg and M.A. Readdy, Sheffer posets and $r$-signed permutations, Ann. Sci. Math. Québec 19 (1995), 173-196. MR1365825 (96m:06005)

[5] R. Ehrenborg and M.A. Readdy, The $r$-cubical lattice and a generalization of the $c d$-index, Europ. J. Combin. 17 (1996), 709-725. MR 1415312 (97k:06014)

[6] G.H. Hardy, Ramanujan, Twelve lectures on subjects suggested by his life and work, New York, Chelsea Publishing Company, 1959. MR0106147 (21:4881)

[7] M.E. Hoffman, Derivative polynomials, Euler polynomials, and associated integer sequences, Electron. J. Combin. 6 (1999), R21. MR1685701 (2000c:11027)

[8] K. Ireland and M. Rosen, A Classical Introduction to Modern Number Theory, SpringerVerlag, 1990 MR1070716 (92e:11001)

[9] E. Landau, Elementary Number Theory, New York, Chelsea Publishing Company, 1958. MR0092794(19:1159d)

[10] N. Metropolis, G.-C. Rota, V. Strehl and N. White, Partitions into chains of a class of partially ordered sets, Proc. Amer. Math. Soc.71 (1978), 193-196. MR0551483 (58:27667)

[11] M.B. Nathanson, Elementary Methods in Number Theory, Springer-Verlag, 2000. MR.1732941 (2001j:11001)

[12] G. Pall, The distribution of integers represented by binary quadratic forms, Bull. Amer. Math. Soc. 49 (1943), 447-449. MR0008084(4:240g)

[13] M. Purtill, André permutations, lexicographic shellability and the $c d$-index of a convex polytope, Trans. Amer. Math. Soc. 338 (1993), 77-104. MR:1094560 (93j:52017)

[14] D. Shanks, On the conjecture of Hardy and Littlewood concerning the number of primes of the form $n^{2}+a$, Math. Comp. 14 (1960), 321-332. MR0120203 (22:10960)

[15] D. Shanks and J.W. Wrench, The calculation of certain Dirichlet series, Math. Comp. 17 (1963), 136-154. MR0159796(28:3012)

[16] D. Shanks, Polylogarithms, Dirichlet series, and certain constants, Math. Comp. 18 (1964), 322-324. MR0175275(30:5460)

[17] D. Shanks, Generalized Euler and class numbers, Math. Comp. 21 (1967), 689-694; Corrigenda, ibid, 22 (1968), 699. MR0223295(36:6343) 
[18] D. Shanks, Calculation and application of Epstein zeta functions, Math. Comp. 29 (1975), 271-287. MR0409357(53:13114a)

[19] N.J.A. Sloane, The On-Line Encyclopedia of Integer Sequences, http://www.research. att.com/ ${ }^{\sim}$ njas/sequences.

[20] T.A. Springer, Remarks on a combinatorial problem, Nieuw Arch. Wisk. 19 (1971), 30-36. MR0338199(49:2965)

[21] R.P. Stanley, Binomial posets, Möbius inversion, and permutation enumeration, J. Combin. Theory Ser. A 20 (1976), 336-356. MR0409206 (53:12968)

Center for Combinatorics, LPMC-TJKlC, Nankai University, Tianjin 300071, PeoPle's RePublic of China

E-mail address: chen@nankai.edu.cn

Center for Combinatorics, LPMC-TJklC, Nankai University, Tianjin 300071, PeoPLE'S RePUBlic OF China

E-mail address: fjy@cfc.nankai.edu.cn

Center for Combinatorics, LPMC-TJKLC, Nankai University, Tianjin 300071, PeoPle's Republic of ChinA

E-mail address: jyt@cfc.nankai.edu.cn 